\title{
Das Tricarbonyl-eisen-cycloheptatrienid-Anion $\left[\mathrm{C}_{7} \mathrm{H}_{7} \mathrm{Fe}(\mathrm{CO})_{3}\right]^{-}$: $\eta^{3}$-Allylanion- oder $\eta^{4}$-Butadienkomplex?
}

The Tricarbonyl Iron Cycloheptatrienide Anion: $\eta^{3}$-Allyl Anion or $\eta^{4}$-Butadiene Complex ?

Peter Hofmann

Institut für Organische Chemie der Universität Erlangen-Nürnberg

Z. Naturforsch. 33 b, 25I-260 (1978); eingegangen am 19. Dezember 1977

Tricarbonyl Iron Cycloheptatrienide, Fluxionality, Ground State Structure, Molecular Orbital Calculations

Molecular orbital calculations for $\left[\mathrm{C}_{7} \mathrm{H}_{7} \mathrm{Fe}(\mathrm{CO})_{3}\right]^{-}$predict a $\eta^{3}$-allyl anion $\mathrm{Fe}(\mathrm{CO})_{3} \mathrm{com}$ plex with un-coordinated diene part of the $\mathrm{C}_{7} \mathrm{H}_{7}$ ligand to be more stable than the alternative $\eta^{4}$-butadiene $\mathrm{Fe}(\mathrm{CO})_{3}$ form, the cyclopolyenyl ligand being nonplanar in either case. The tiny energy difference between both coordination modes accounts for the observed high fluxionality of the anion.

Das instabile, hochreaktive CycloheptatrienidAnion $\mathrm{C}_{7} \mathrm{H}_{7}-$ findet als monocyclisches, potentiell antiaromatisches $8 \pi$-System seit langem Interesse in der organischen Chemie [1]. Wie bei zahlreichen anderen Beispielen unbeständiger Moleküle [2] war es kürzlich auch im Falle des $\mathrm{C}_{7} \mathrm{H}_{7}$--Ions möglich, dieses Teilchen in der Ligandensphäre eines Übergangsmetalls zu erzeugen und im relativ beständigen

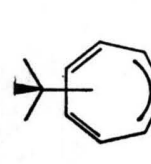

$1 \mathrm{~A}$

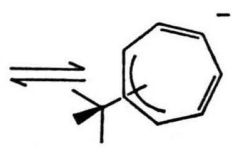

18

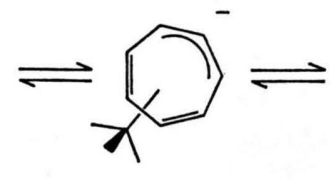

$1 \mathrm{~A}$
Tricarbonylkomplex $\mathrm{Na}^{+}\left[\mathrm{C}_{7} \mathrm{H}_{7} \mathrm{Fe}(\mathrm{CO})_{3}\right]^{-}$zu stabilisieren [3]. Die Chemie des ungewöhnlichen Cyclopolyenylkomplex-Anions $\left[\mathrm{C}_{7} \mathrm{H}_{7} \mathrm{Fe}(\mathrm{CO})_{3}\right]^{-}(\mathbf{1})$ wird zur Zeit intensiv untersucht [4], insbesondere seit es Behrens und Mitarbeitern gelang, nicht nur das Natriumsalz in analysenreiner Form leicht zugänglich zu machen, sondern auch stabile, kristalline Salze von 1 mit anderen geeigneten Kationen darzustellen [5].

$$
\frac{1}{-}=\mathrm{Fe}(\mathrm{CO})_{3}
$$

Anion 1 zeigt NMR-spektroskopisch nachweisbar typisch fluktuierendes Verhalten (Nonrigidity); im Gegensatz zu den bekannten isoelektronischen Fällen des $\mathrm{C}_{8} \mathrm{H}_{8} \mathrm{Fe}(\mathrm{CO})_{3}[6]$ und $\mathrm{C}_{7} \mathrm{H}_{7} \mathrm{Co}(\mathrm{CO})_{3}$ [7] gelang es jedoch bisher nicht, die dynamischen, intramolekularen Prozesse, die hier wie dort für die beobachtete Äquivalenz aller CH-Gruppen einerseits und aller drei Carbonylliganden andererseits verantwortlich sind, bei tiefer Temperatur einzufrieren. In Analogie zum $\mathrm{C}_{8} \mathrm{H}_{8} \mathrm{Fe}(\mathrm{CO})_{3}$ kann man sicherlich davon ausgehen, daß, abgesehen von der extrem leicht möglichen $\mathrm{Fe}(\mathrm{CO})_{3}$-Rotation, haptotrope 1.2-Verschiebungen [8] des Metalltricarbonylrestes relativ zum begleitend relaxierenden Cyclopolyenylligan-

Sonderdruckanforderungen an Dr. P. Hofmann, Institut für Organische Chemie I der Universität ErlangenNürnberg, Henkestraße 42, D.8520 Erlangen. den die Ursache des fluktuierenden Verhaltens von 1 sind. Die Frage nach der energieärmsten Struktur von 1 und somit nach dem Minimum im Energieprofil des entarteten Verschiebungsprozesses blieb aber zunächst offen [9].

Da dem detaillierten Verständnis von Mechanismen und Energetik haptotroper Verschiebungen in Organoübergangsmetallkomplexen, insbesondere in sog. ,,ring whizzer"-Systemen [10] momentan besonderes Interesse zukommt, wurden im Rahmen einer theoretischen Untersuchung solcher Phänomene auch für 1 MO-Modellrechnungen durchgeführt, die zu interessanten Aussagen über dessen Bindungsverhältnisse führten und über die hier berichtet werden soll.

Im Einklang mit der zu fordernden Kryptonkonfiguration am Eisen stehen dem Ion 1 a priori 
zwei strukturelle Alternativen als Energieminima offen:

a) Die Struktur eines $\eta^{4}$-Butadienkomplexes mit unkomplexiertem Allylanion-Teil (Struktur 1A). Dies entspricht den Verhältnissen $\mathrm{im}_{8} \mathrm{H}_{8} \mathrm{Fe}(\mathrm{CO})_{3}$ [11] und damit dem üblichen Bindungstyp der Vielzahl bekannter, stabiler Dien- $\mathrm{Fe}(\mathrm{CO})_{3}$-Komplexe und stellt wohl die intuitiv erwartete Grundzustandsgeometrie für 1 dar.

b) Die Form eines $\eta^{3}$-Allylanionkomplexes $1 \mathrm{~B}$ mit nicht ans Metall gebundenem Butadiensegment im Siebenringliganden. Diese Geometrie entspricht dem in Allyl-Co(CO) $)_{3}$-Komplexen [12] (und wahrscheinlich auch im zu l isoelektronischen $\left.\mathrm{C}_{7} \mathrm{H}_{7} \mathrm{Co}(\mathrm{CO})_{3}\right)$ [7] vorliegenden Strukturtyp.
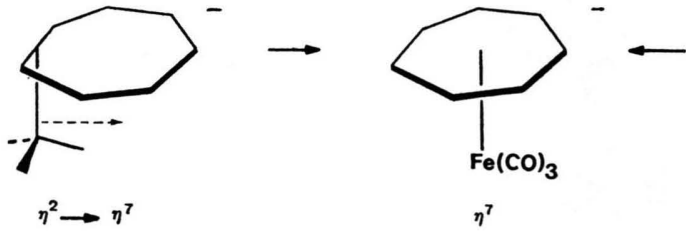

2
Der relative Energieinhalt von $\eta^{4}$ - und $\eta^{3}$-Form 1 A bzw. 1 B, die beide während der entarteten Umlagerung von 1 durchlaufen werden müssen, spielt eine entscheidende Rolle für die Energiebarriere dieses Vorgangs.

Den durchgeführten Molekülorbital-Berechnungen vom Extended Hückel-Typ (siehe Anhang) und einer Analyse der Wechselwirkungen zwischen $\mathrm{Fe}(\mathrm{CO})_{3}$-Fragment und dem Liganden $\mathrm{C}_{7} \mathrm{H}_{7}$ - für beide zur Diskussion stehende Alternativstrukturen $1 \mathrm{~A}$ und $1 \mathrm{~B}$ wurde zunächst ein geometrisch stark vereinfachtes Modell 2 mit planarem $\mathrm{C}_{7} \mathrm{H}_{7}{ }^{-}$-Liganden (lokale $\mathrm{D}_{7 \mathrm{~h}}$-Symmetrie) und $\mathrm{C}_{3 \mathrm{v}}$-symmetrischem $\mathrm{Fe}(\mathrm{CO})_{3}$-Rest (alle Winkel $\mathrm{CO}-\mathrm{Fe}-\mathrm{CO}=90^{\circ}$, Oktaederfragment) zugrunde gelegt.
Abb. 1 gibt das berechnete Energieprofil für eine willkürlich in 1,8 $\AA$ Abstand äquidistant zur Ringebene und in der Spiegelebene des Systems erfolgende haptotrope Verschiebung des Metallrestes wieder, in deren Verlauf sich die $\mathrm{Fe}(\mathrm{CO})_{3}$-Gruppe unterhalb des Rings aus der $\eta^{2}$ - über die $\eta^{4}$ - und aus $\operatorname{der} \eta^{1}$ - über die $\eta^{3}$-Position heraus auf die Ringmitte $\left(\eta^{7}\right.$-Struktur) zubewegt. Der Metalltricarbonylrest ist dabei jeweils so orientiert, daß für die Minima in $\eta^{4}$ - und $\eta^{3}$-Stellung die energetisch günstigere ,,gestaffelte" Konformation [13] des $\mathrm{Fe}(\mathrm{CO})_{3}$-Rotors relativ zum gebundenen Butadien- oder Allylteil vorliegt.

Überraschenderweise ist für unser Modell das auf dem Weg $\eta^{1} \rightarrow \eta^{7}$ durchlaufene Minimum, welches dem Allylanionkomplex 1 B entspricht, geringfügig energieärmer als das von der $\eta^{2}$-Position aus erreichte Minimum entsprechend $\mathbf{1 A}$. Auch für veränderten Metall-Ring-Abstand bleibt diese Charakteristik des Energieprofils der beschriebenen Modellbewegung des $\mathrm{Fe}(\mathrm{CO})_{3}$-Fragments bestehen. Annäherung der Geometrie an die $\eta^{7}$-Struktur (formal über $\eta^{6}$ - bzw. $\eta^{5}$-Zwischenstationen) führt erwartungsgemäß zu extremem Energieanstieg, worauf später noch kurz eingegangen werden soll.
Die wohlbeschriebenen und ausführlich diskutierten Valenzorbitale eines $\mathrm{Fe}(\mathrm{CO})_{3}$-Fragments $\left(\mathrm{d}^{8}\right)[14]$ und deren Wechselwirkung mit den $\pi$-MO's eines $\mathrm{C}_{7} \mathrm{H}_{7}$-Anions [15] bieten eine einfache Möglichkeit, Elektronenstruktur und geometrische Präferenzen im $\left[\mathrm{C}_{7} \mathrm{H}_{7} \mathrm{Fe}(\mathrm{CO})_{3}\right]^{-} \mathrm{zu}$ beschreiben.

Abb. 2 zeigt das aus berechneten Konturdiagrammen abgeleitete Aussehen [14] sowie die relativen Energien der $\mathrm{Fe}(\mathrm{CO})_{3}$-Valenz-MO's sowie die vertrauten $\pi$-MO's des $\mathrm{C}_{7} \mathrm{H}_{7}{ }^{-}$.

Für das $\mathrm{Fe}(\mathrm{CO})_{3}$-System finden sich neben einem bei relativ hoher Energie gelegenen rotationssymmetrischen Akzeptororbital ( $2 \mathrm{a}_{1}$, Hybridorbital mit hauptsächlich s- und z-Charakter) [16] die 5 nächsttieferen, vorwiegend d-Anteil aufweisenden Niveaus aufgespalten in einen tiefliegenden Satz von 3 MO's $\left(1 a_{1}, l e\right)$, abgeleitet vom $t_{2 g}$-Niveau eines $M(C O)_{6}$ Oktaeders, und in ein höherliegendes, entartetes Orbital (2e). Im gewählten Koordinatensystem ist $1 \mathrm{a}_{1} \mathrm{zu}$ beschreiben als $z^{2}$ am Metall, bindend wechselwirkend mit $\pi^{*}$-Niveaus der Carbonylgruppen, während die beiden Komponenten des $1 \mathrm{e}$ Satzes, $1 e_{s}$ und $l e_{a}$, zur Hauptsache $x^{2}-y^{2}$ - bzw. xyCharakter aufweisen. $1 \mathrm{a}_{1}$ und $1 \mathrm{e}$ können quasi als freie Elektronenpaare am Eisen aufgefaßt werden. 


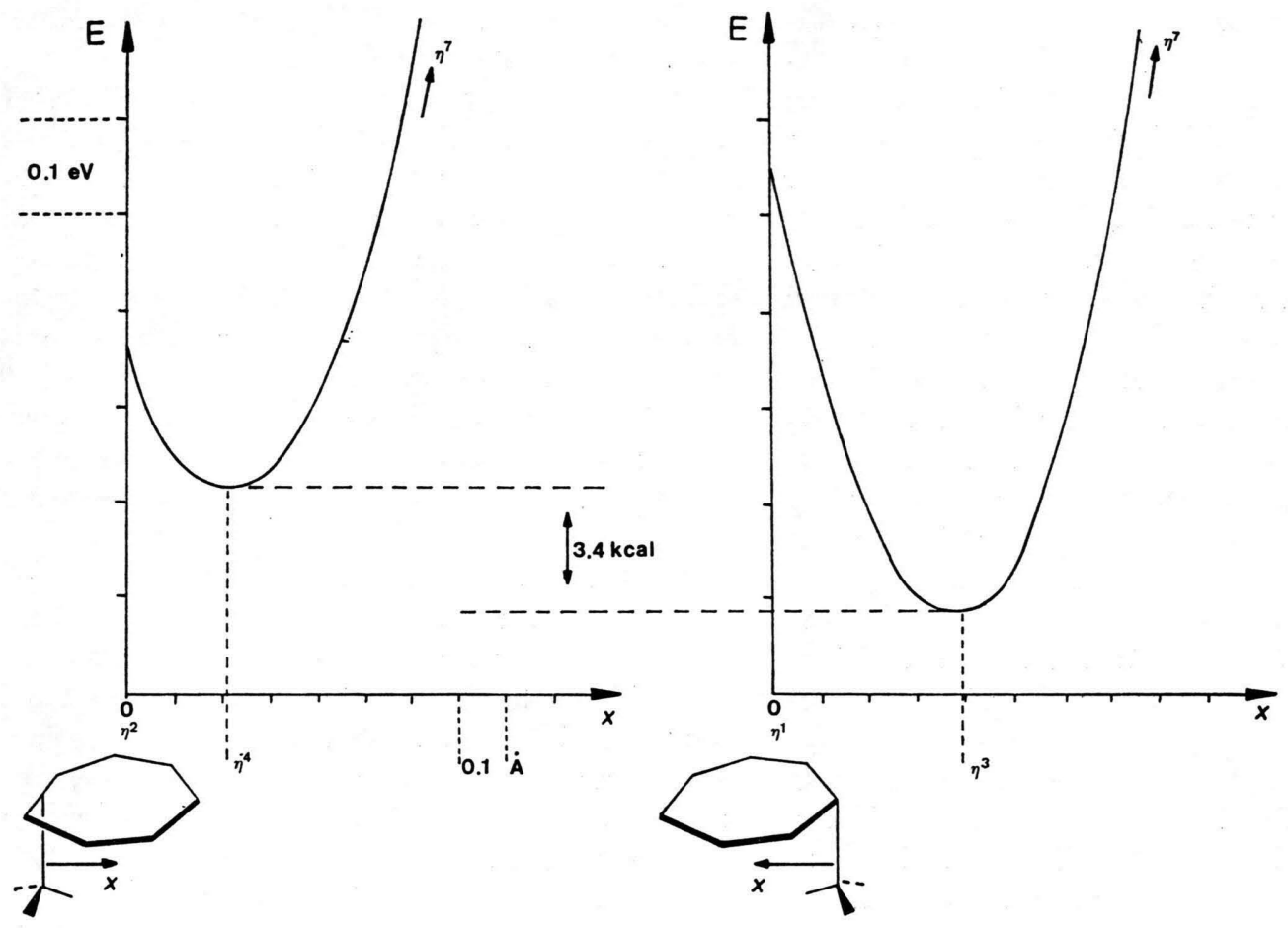

Abb. 1.


Abb. 2. 
Im $2 \mathrm{e}$-Niveau hingegen überwiegt für $2 \mathrm{e}_{\mathrm{s}}$ der $\mathrm{xz}$ bzw. für $2 \mathrm{e}_{\mathrm{a}}$ der yz-Anteil am Metallzentrum, $\mathrm{x}$ und y-Funktion des Metalls mischt hier bindend ein, Schwächung der Antibindung zu den CO-Liganden und Rehybridisierung hin auf potentielle Partner des $\mathrm{Fe}(\mathrm{CO})_{3}$-Fragments sind die Folgen. Ein entscheidender Punkt liegt darin, daß in der $\mathrm{C}_{3 \mathrm{v}^{-}}$ Symmetrie des Metalltricarbonylrestes $x^{2}-y^{2}$ und $\mathrm{xy}$ einerseits sowie $\mathrm{xz}$ und $\mathrm{yz}$ andererseits vom gleichen Symmetrietyp sind. Dies führt zur in den Darstellungen für $1 \mathrm{e}$ und $2 \mathrm{e}$ angedeuteten RechtsLinks-Asymmetrie der Komponenten dieser ValenzMO's, da infolge bindender Einmischung von $x \mathrm{z}$ und $\mathrm{y} z$ in $1 \mathrm{e}$ und infolge antibindender Einmischung vom $x^{2}-y^{2}$ und $x y$ in $2 e$ eine Intra-FragmentPolarisierung auftritt, die die $\pi$-Bindung zu den Carbonylgruppen für le stärkt (d.h. le stabilisiert) und die $2 \mathrm{e}$ bei relativ hoher Energie erscheinen läßt.

Die $\pi$-MO's des planaren $\mathrm{C}_{7} \mathrm{H}_{7}$ - in Abb. 2 sind in der üblichen Weise dargestellt, die relative Koeffizientendichte in den einzelnen Niveaus ist durch die Größe der Kreise angedeutet und kann im Rahmen üblicher Störungsargumente [15] leicht verstanden werden, wenn man sich $\mathrm{C}_{7} \mathrm{H}_{7}-$ z.B. aus Butadienund Allylteil entstanden denkt.

Sowohl Struktur 1A als auch 1 B des Anions 1 sind bezüglich der Wechselwirkungen der beschriebenen $\mathrm{Fe}(\mathrm{CO})_{3}-$ und $\mathrm{C}_{7} \mathrm{H}_{7}-\mathrm{MO}$ 's dadurch ausgezeichnet, daß das bei $\eta^{7}$-Geometrie relativ zum Metall pseudo- $\delta$-symmetrische, doppelt besetzte und entartete $3 \pi$-Niveau $\left(3 \pi_{\mathrm{s}}\right.$ und $\left.3 \pi_{\mathrm{a}}\right)$ des Cyclopolyenylsystems, welches in der „PianostuhlStruktur" von $1 e_{s}\left(x^{2}-y^{2}\right)$ und $1 e_{a}(x y)$ destabilisiert wird, in der nicht zentrosymmetrischen Geometrie mit dem relativ zum Ring $\pi$-symmetrischen, ebenfalls doppelt besetzten 2e-Satz in Wechselwirkung treten kann.

Die charakteristischen Unterschiede zwischen $\eta^{7}$ und z.B. $\eta^{3}$-Koordination sind qualitativ anhand der Wechselwirkungsdiagramme in Abb. 3 zu ersehen.

Die bindenden Wechselwirkungen zwischen $3 \pi$ und $2 \mathrm{e}$, die bei $\eta^{3}$ - und $\eta^{4}$-Struktur auftreten, gehen bei weiterer Verschiebung des $\mathrm{Fe}(\mathrm{CO})_{3}$-Restes auf die Ringmitte hin rasch verloren, die einsetzende Wechselwirkung zwischen $1 \mathrm{e}$ und $3 \pi$ aber destabilisiert die beiden höchsten besetzten Niveaus stark, bis schließlich im „Pianostuhl-Fall“ diese Orbitale

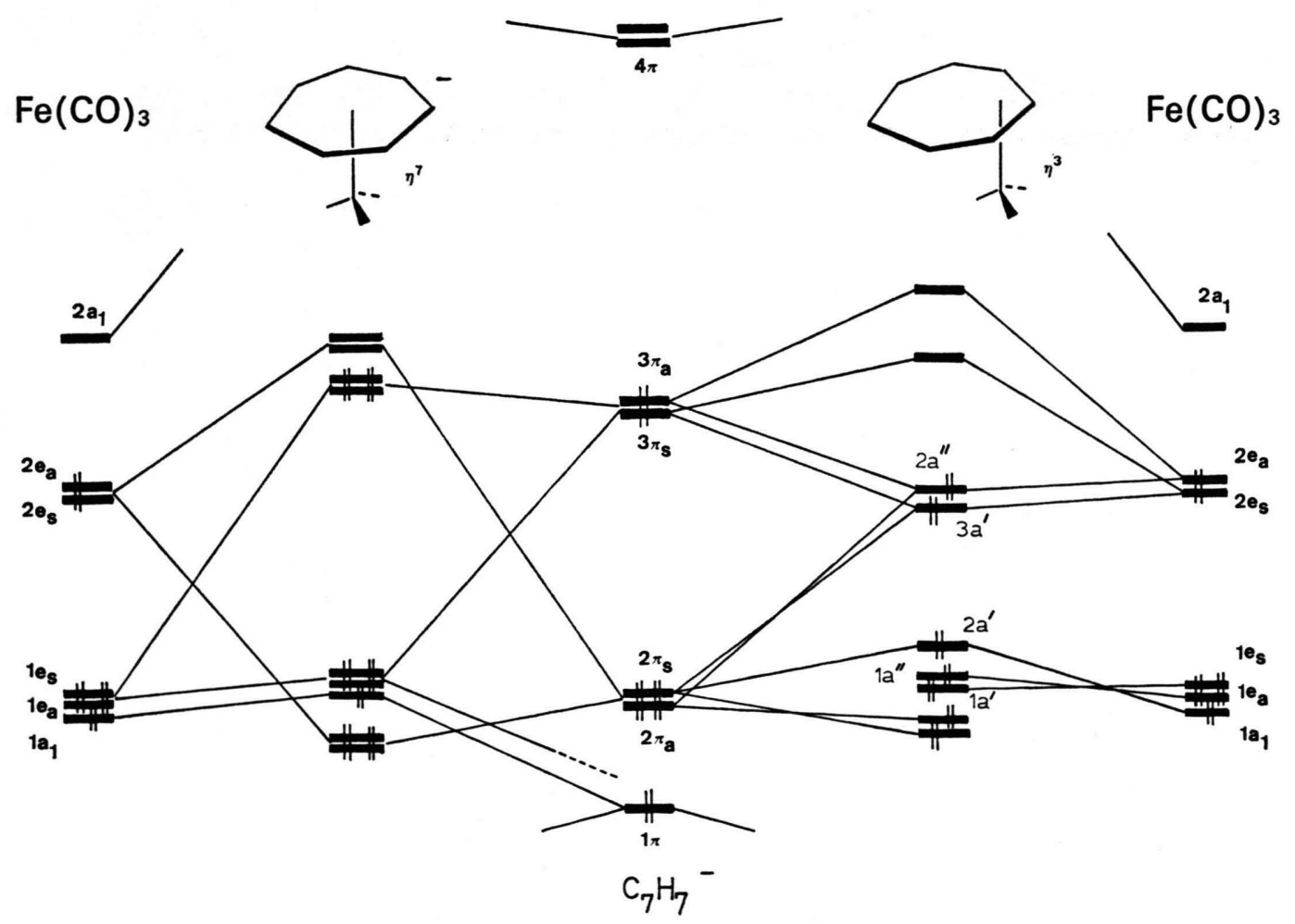

Abb. 3. 
als antibindende Kombinationen von $3 \pi$ mit le und mit vorwiegend Ring-Charakter bei so hoher Energie erscheinen, da $\beta$ die in der $\eta^{7}$-Geometrie mögliche Absenkung von $2 \pi$ durch $2 \mathrm{e}$ demgegenüber keine Rolle mehr spielt. Im Einklang mit der Edelgasregel ist ein Umlagerungsmechanismus für Anion 1, der die $\eta^{7}$-Koordination einbezieht, energetisch sicherlich unerreichbar. Aber auch für analoge Systeme mit zwei Elektronen weniger, etwa für $\mathrm{C}_{8} \mathrm{H}_{8} \mathrm{Cr}(\mathrm{CO})_{3}, \quad \mathrm{C}_{7} \mathrm{H}_{7} \mathrm{Fe}(\mathrm{CO})_{3}{ }^{+}$oder $\mathrm{C}_{7} \mathrm{H}_{7} \mathrm{Mn}(\mathrm{CO})_{3}$ dürfte aus theoretischer Sicht wegen der extrem ungünstigen Energie der noch in den praktisch entarteten destabilisierten $3 \pi$-Niveaus verbleibenden Elektronen der von Cotton auf Grund von NMRDaten postulierte ,,random shift“ über eine „Piano-
stuhl"-Zwischenstufe [17] kaum realistisch sein [18], neuere experimentelle Untersuchungen von Whitesides scheinen dies zu belegen [10].

Für um vier Elektronen ärmere Komplexe wie $\mathrm{C}_{7} \mathrm{H}_{7} \mathrm{Cr}(\mathrm{CO})_{3}{ }^{+}$oder $\mathrm{C}_{7} \mathrm{H}_{7} \mathrm{~V}(\mathrm{CO})_{3}[19]$ ist die $\eta^{7}$-Form selbstverständlich Grundzustandsgeometrie.

Die im Energieprofil von Abb. 1 dargestellte schwache Bevorzugung der $\eta^{3}$-Struktur 1 B über 1 A läßt sich anhand der geringfügig unterschiedlichen Wechselwirkungen der angeführten $\mathrm{Fe}(\mathrm{CO})_{3}$-Fragmentorbitale mit dem Siebenring verstehen. Abb. 4 zeigt die berechnete Energie der 5 höchsten besetzten Einelektronenniveaus für die bereits bezüglich der Gesamtenergie in Abb. 1 gezeigten $\mathrm{Fe}(\mathrm{CO})_{3-}$ Verschiebungen $\eta^{1} \rightarrow \eta^{3}$ und $\eta^{2} \rightarrow \eta^{4}$.
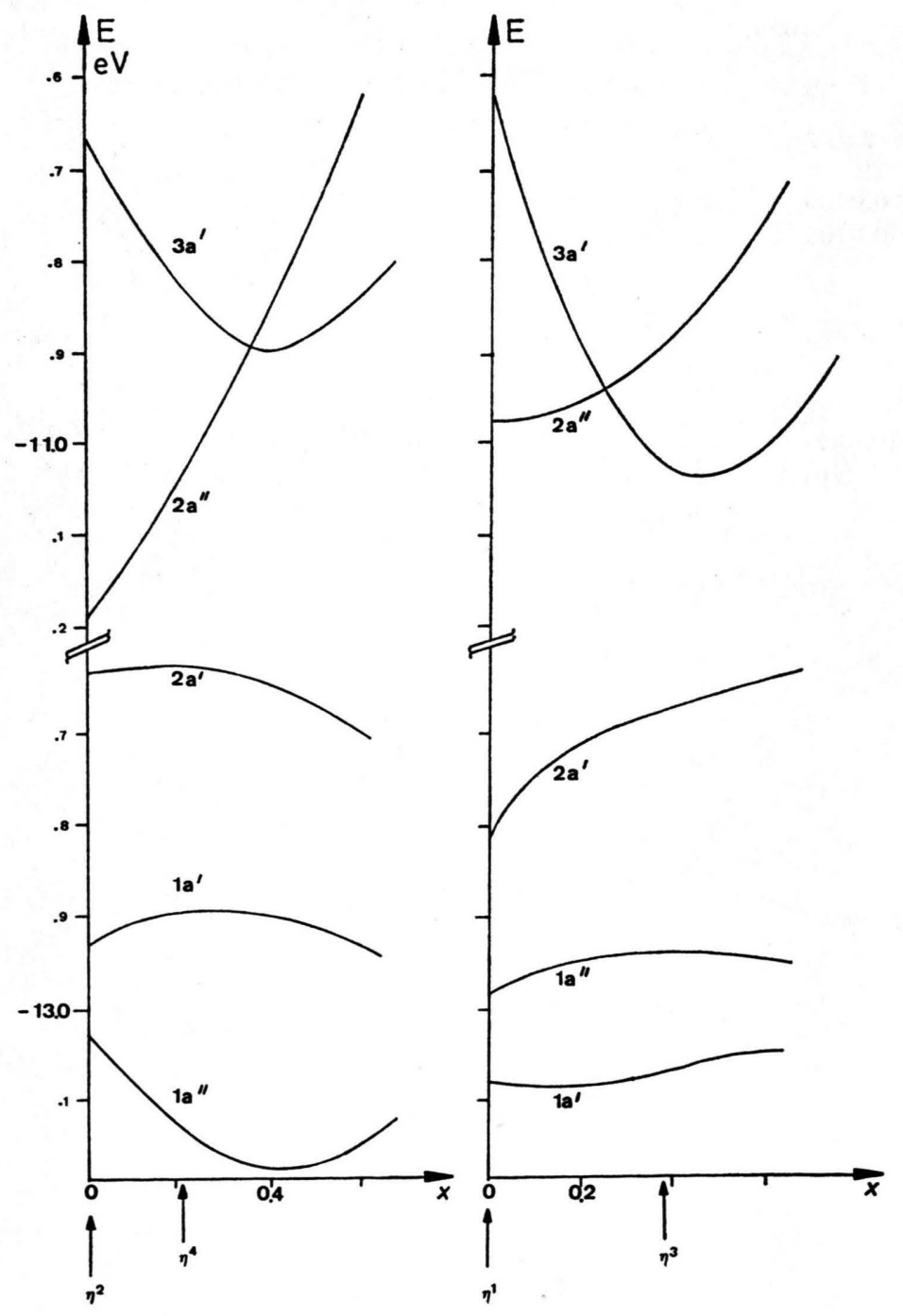

Abb. 4. 
Betrachtet man allein die gezeigten 5 höchsten besetzten MO's, die die dominierenden $\mathrm{Fe}(\mathrm{CO})_{3} / \mathrm{C}_{7} \mathrm{H}_{7}{ }^{-}$Wechselwirkungen wiedergeben, so ist die Energie für das $\eta^{3}$-Minimum in der Rechnung um $4,2 \mathrm{kcal}$ günstiger, alle tiefer liegenden Niveaus schwächen diese Präferenz gegenüber der $\eta^{4}$-Geometrie zwar ab, heben sie aber nicht auf. Der Hauptbeitrag zur Ausbildung beider Minima $\mathbf{1 A}$ und $\mathbf{1 B}$ auf dem Weg $\eta^{1} \rightarrow \eta^{7}$ bzw. $\eta^{2} \rightarrow \eta^{7}$ findet sich in der energetischen Absenkung der bindenden Kombination von $2 \mathrm{e}_{\mathrm{s}}$ mit $3 \pi_{\mathbf{s}}\left(3 a^{\prime}\right.$ in Abb. 3). Die Stabilisierung dieses MO's ist jedoch im $\eta^{3}$-Fall wesentlich ausgeprägter als auf dem Wege zum $\eta^{4}$-Zustand, was sich in den $\left\langle 3 \pi_{\mathrm{s}} / 2 \mathrm{e}_{\mathrm{s}}\right\rangle$-Gruppenüberlappungen für Form $\mathbf{1 A}$ und $1 \mathrm{~B}$ in Tab. I wiederspiegelt.

Tab. I. Relevante Gruppenüberlappungen zwischen $\mathrm{Fe}(\mathrm{CO})_{3}$ - und $\mathrm{C}_{7} \mathrm{H}_{7}-$-Fragmentorbitalen für $\eta^{3}$ - und $\eta^{4}$-Minimum.

\begin{tabular}{lllll}
\hline & $\left\langle 3 \pi_{\mathrm{s}} / 2 \mathrm{e}_{\mathrm{s}}\right\rangle$ & $\left\langle 3 \pi_{\mathrm{a}} / 2 \Theta_{\mathrm{a}}\right\rangle$ & $\left\langle 2 \pi_{\mathrm{a}} / 2 \mathrm{e}_{\mathrm{a}}\right\rangle$ & $\left\langle 2 \pi_{\mathrm{s}} / 2 \Theta_{\mathrm{s}}\right\rangle$ \\
\hline $1 \mathrm{~A}$ & 0,0788 & 0,1613 & 0,1690 & 0,0496 \\
$1 \mathrm{~B}$ & 0,1049 & 0,1462 & 0,1720 & 0,0708 \\
\hline
\end{tabular}

Räumliche Ausdehnung und Richtungscharakter von $2 \mathrm{e}_{\mathrm{s}}$ und das Aussehen des $3 \pi_{\mathrm{s}}$-Orbitals im Ring (nodale Eigenschaften und Koeffizientendichteverteilung) sorgen für die bessere Überlappung bei Geometrie 1 B verglichen mit $1 \mathrm{~A}$. Dies ist in 3 und 4 schematisch wiedergegeben.

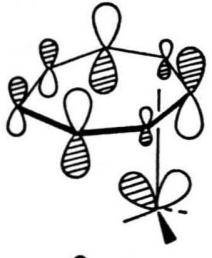

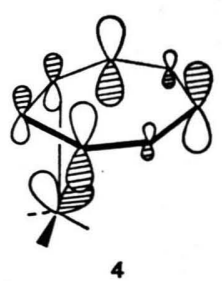

Betrachten wir die antisymmetrischen Komponenten von $3 \pi$ und $2 \mathrm{e}$, so sind die $\left\langle 3 \pi_{\mathrm{a}} / 2 \mathrm{e}_{\mathrm{a}}\right\rangle$-Überlappungen für den $\eta^{2}$-Startpunkt und das $\eta^{4}$ Minimum $1 \mathrm{~A}$ zwar größer als für die $\eta^{1}$-Position und das $\eta^{3}$-Minimum 1 B, die Abnahme der Wechselwirkungen zwischen $3 \pi_{\mathrm{a}}$ und $2 \mathrm{e}_{\mathrm{a}}$ jedoch, d.h. die Destabilisierung des $2 \mathrm{a}^{\prime \prime}$-Niveaus in Abb. 4, ist für die $\eta^{2} \rightarrow \eta^{4}$-Verschiebung wesentlich drastischer (vgl. 5 und 6), und wirkt hier der an sich schon schwächeren Stabilisierung von $3 a^{\prime}$ entgegen.
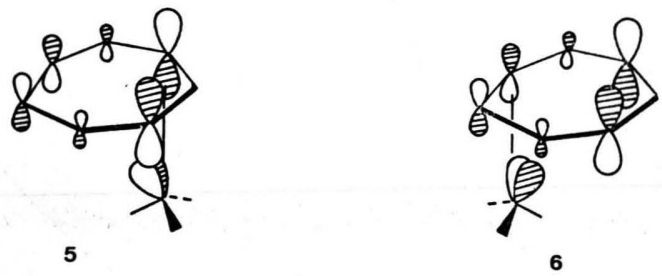

Die entscheidenden Unterschiede für die Minima $1 \mathrm{~A}$ und $\mathbf{1 B}$ des verwendeten Modells sind in Abb. 5 deutlich herausgestellt.

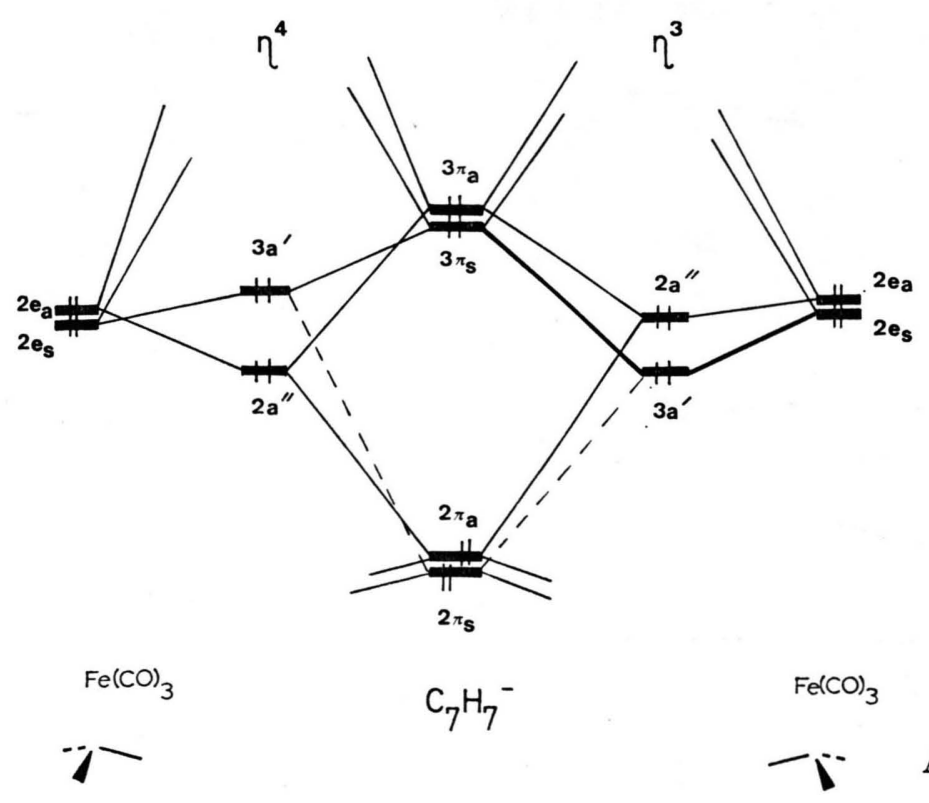

Abb. 5. 
Das im $\eta^{3}$-Fall energiegünstigere $3 \mathrm{a}^{\prime}$-Orbital besitzt sowohl für $\eta^{3}$ - als auch für $\eta^{4}$-Strukturmodell $\mathrm{zu}$ je $44 \%$ Cycloheptatrienid-Anteil, ist also in beiden Fällen mehr am $\mathrm{Fe}(\mathrm{CO})_{3}$-Fragment lokalisiert. $3 \mathrm{a}^{\prime}$ verursacht zwar zusammen mit der etwas günstigeren Energiebilanz der nächsttieferen MO's, welche die destabilisierenden Vierelektronenwechselwirkungen zwischen Eisen-d-Elektronenpaaren und $1 \pi, 2 \pi$ sowie $\sigma$-MO's des $\mathrm{C}_{7} \mathrm{H}_{7}-$ repräsentieren, die Bevorzugung des $\eta^{3}$-Bindungsmodus, es ist jedoch das $2 a^{\prime \prime}$-Niveau des Komplexes, das bezüglich der Ladungsverteilung und Bindungsverhältnisse in $1 \mathrm{~A}$ und $\mathbf{1 B}$ zu Unterschieden führt.

Wie Tab. I zu entnehmen ist, übertrifft nämlich die $\left\langle 2 \pi_{\mathrm{a}} / 2 \mathrm{e}_{\mathrm{a}}\right\rangle$-Überlappung (insbesondere für den $\eta^{3}$-Fall) diejenige zwischen $3 \pi_{\mathrm{a}}$ und $2 \mathrm{e}_{\mathrm{a}}$. Deshalb $\mathrm{mu} \beta$ ins 2a"-Orbital auch ein gewisser Anteil an $2 \pi_{\mathrm{a}}$-Charakter antibindend einmischen. Diese antibindende Beteiligung von $2 \pi_{\mathrm{a}}$, die für die Geometrie $1 \mathrm{~B}$ besonders stark ist, hält das $2 \mathrm{a}^{\prime \prime}$-MO bei höherer Energie als es ohne diese Wechselwirkung der Fall wäre und lokalisiert es zum überwiegenden Teil im Liganden $\mathrm{C}_{7} \mathrm{H}_{7}-$.

Dabei resultiert für die $\eta^{3}$-Struktur (kleinere $\left\langle 3 \pi_{\mathrm{a}} / 2 \mathrm{e}_{\mathrm{a}}\right\rangle$ - und größere $\left\langle 2 \pi_{\mathrm{a}} / 2 \mathrm{e}_{\mathrm{a}}\right\rangle$-Überlappung) ein energetisch ungünstigeres und stärker am Ringsystem lokalisiertes $2 \mathrm{a}^{\prime \prime}$-MO als für den $\eta^{4}$-Fall (bessere $\left\langle 3 \pi_{\mathrm{a}} / 2 \mathrm{e}_{\mathrm{a}}\right\rangle$ - und geringere $\left\langle 2 \pi_{\mathrm{a}} / 2 \mathrm{e}_{\mathrm{a}}\right\rangle$-Überlappungsterme). Zudem führt die jeweils antibindende Einmischung von $2 \pi_{\mathrm{a}}$ in $2 \mathrm{a}^{\prime \prime}$ zum in $\mathbf{7}$ und 8 dargestellten Aussehen dieser Wellenfunktionen für $\eta^{3}$ - und $\eta^{4}$-Geometrie.
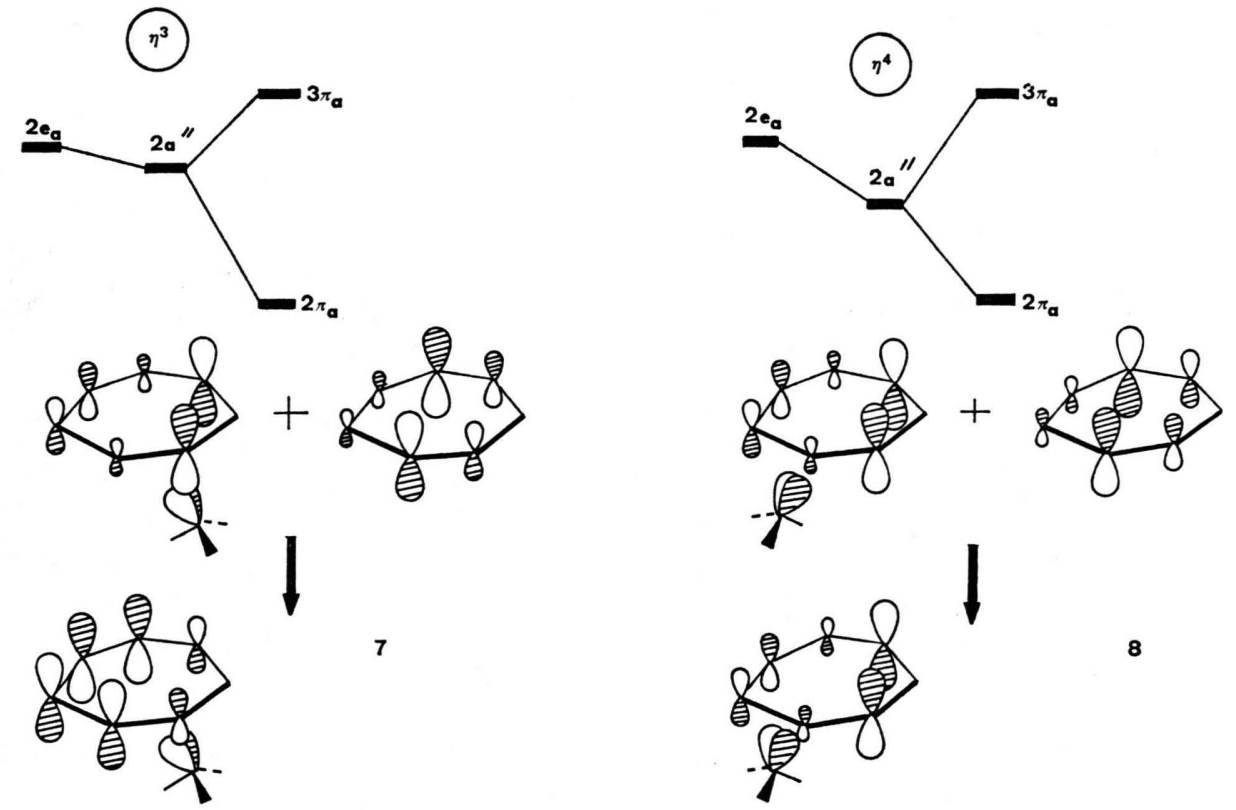

Die für $1 \mathbf{B}$ etwas stärkere Konzentration des in 7 dargestellten Niveaus $2 \mathrm{a}^{\prime \prime}$ im $\mathrm{C}_{7} \mathrm{H}_{7}{ }^{-}$-System $(66 \%$ gegenüber $59 \%$ bei $\mathbf{1 A}$ ) führt interessanterweise dazu, daß der formal einem Allylanion-Komplex entsprechende Strukturtyp 1 B nach der Rechnung weniger negative Ladung am Eisen aufweist als das Modell $1 \mathrm{~A}(-0,199$ gegenüber - 0,241), oder anders ausgedrückt, mehr Ladung am Ringliganden läßt.

Außerdem hat die Einmischung von $2 \pi_{\mathrm{a}}$ ins $2 \mathrm{a}^{\prime \prime}$ Niveau entsprechend 7 und 8 zur Folge, daß die $2 \mathrm{a}^{\prime \prime}$-Wellenfunktion bei $\eta^{3}$-Koordination überwiegend den Charakter eines Butadien-HOMO's im nichtkoordinierten $\mathrm{C}_{4}$-Teil des Cycloheptatrienidrings annimmt, während sie für die $\eta^{4}$-Struktur als HOMO des nicht ans Eisen gebundenen Allylsegments erscheint. Für die energetisch günstigere $\eta^{3}$-Form $1 \mathrm{~B}$ ergibt sich aus der Besetzung von $2 \mathrm{a}^{\prime \prime}$ mit 2 Elektronen die Konsequenz, daß im Butadienteil des Carbocyclus Bindungsalternanz wie im Butadien vorzufinden sein sollte. Die für 1B errechneten Mulliken-Überlappungspopulationen in 9 geben diesen Trend wieder. Die Bindungen innerhalb des Allylteils und zwischen Allyl- und Butadiensegment sollten demgegenüber länger sein. Inverse 
Verhältnisse, also lange Bindungen im Butadienteil und kurze Bindungsabstände im Allyl- bzw. zwischen Allyl- und Butadiensystem des Siebenrings (vgl. 10) liegen im $\eta^{4}$-Fall vor.

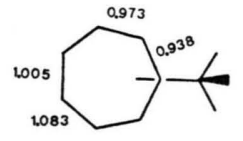

9
10

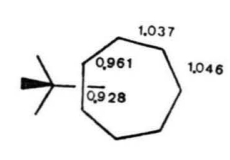

Das in 7 und 8 gezeigte Aussehen des $2 \mathrm{a}^{\prime \prime}$ Orbitals für 1A und $\mathbf{1 B}$ hat weitere Konsequenzen. $2 \mathrm{a}^{\prime \prime}$ ist dasjenige Orbital, das überwiegend im $\mathrm{C}_{7} \mathrm{H}_{7}{ }^{-}$-Ringliganden lokalisiert ist und somit dessen Ladungsverteilung stark beeinflußt. Für die energieärmere $\eta^{3}$-Form sind entsprechend 7 die 2 dieses Niveau besetzenden Elektronen hauptsächlich im Butadienteil des Siebenrings zu erwarten, dessen berechnete Gesamtladungsverteilung 11 bestätigt dies. Es ist wichtig, festzuhalten, daß die Delokalisierung von negativer Ladung in das nichtkoordinierte $\mathrm{C}_{4}$-Fragment von $\mathbf{1 B}$ nicht die Bindungen dort schwächt, weil entsprechend 7 die nodalen Eigenschaften von 2a" denen des HOMO's im Butadien selbst gleichkommen. Für Struktur IA werden laut 8 die beiden $2 \mathrm{a}^{\prime \prime}$-Elektronen das nichtbindende MO des Allylsystems besetzen. Die hierdurch hervorgerufene Gesamtladungsverteilung im Cycloheptatrienidliganden zeigt 12.

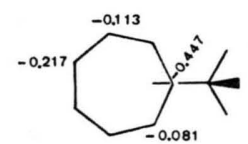

11

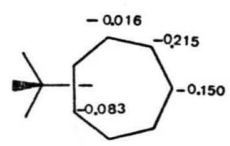

12
Die schematischen Darstellungen der von $2 \mathrm{e}$ und $3 \pi$ abgeleiteten Niveaus $3 a^{\prime}$ und $2 a^{\prime \prime}$ in $3,4,7$ und 8 zeigen ferner deutlich, daß in diesen Niveaus antibindende Wechselwirkungen zwischen Eisen und dem jeweils nicht komplexierten Teil des $\mathrm{C}_{7} \mathrm{H}_{7}{ }^{-}$Systems auftreten. Die berechneten MullikenÜberlappungspopulationen zwischen Fe und „Butadien-Kohlenstoffatomen“ bzw. ,Allyl-Kohlenstoffatomen " in $\mathbf{1 B}$ bzw. $1 \mathbf{A}$ weisen demgemäß negative Werte auf. Dies zeigt an, daß in der Realität für beide Formen eine Relaxation der Ringgeometrie unter Abwinkelung der Butadien- relativ zur Allylebene zu erwarten ist. Test-Rechnungen bestätigen dies: bei gegenüber den Minima in Abb. 1 unveränderter Lage des $\mathrm{Fe}(\mathrm{CO})_{3}$-Restes und bei in sich ebenen Butadien- und Allylsegmenten ergeben sich Abwinkelungen des ungebundenen Ringsegmentes vom Metall weg von $c a .40^{\circ}$. Der Energieunterschied zwischen $\eta^{3}$-Form 13 und $\eta^{4}$-System 14 nimmt dabei zugunsten von 13 noch zu. Ein Kippen des ans Eisen gebundenen Ringteils (wie beim Allylkomplex $\left.\mathrm{C}_{3} \mathrm{H}_{5} \mathrm{Co}(\mathrm{CO})_{3}\right)$ relativ zur Ebene der 3 CarbonylC-Atome (vgl. 13 und 14) erniedrigt die Gesamtenergie weiter, eine vollständige Geometrieoptimierung wurde nicht durchgeführt, da hier einerseits die verwendete Rechenmethode überfordert wäre und da zum anderen die entscheidenden qualitativen Aussagen auch den Berechnungen am planaren Modell 2 zu entnehmen sind.

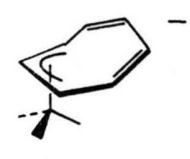

13

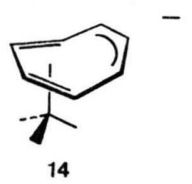

14
Es ist selbstverständlich, daß die hier aufgezeigten Modellrechnungen keine quantitativen Aussagen gestatten. Die zur Hauptsache auf Überlappungsdifferenzen und störungstheoretischen Argumenten zur Wechselwirkung geeigneter Fragment-MO's beruhenden Ergebnisse können jedoch qualitativ richtige Trendaussagen liefern.

Es ist befriedigend, feststellen zu können, daß eine durch die hier geschilderte Vorhersage der $\eta^{3}$. Allylanionstruktur für 1 mitangeregte und inzwischen abgeschlossene Röntgenstrukturanalyse dieses interessanten Übergangsmetallkomplexes, über die in der nachstehenden Publikation berichtet wird, die gemachten Aussagen bestätigt hat [5].

Das dynamische Verhalten von Anion 1 ist nach den geschilderten Ergebnissen als entlang der Peripherie des $\mathrm{C}_{7} \mathrm{H}_{7}-$ verlaufende 1.2-Verschiebung des praktisch frei drehbaren $\mathrm{Fe}(\mathrm{CO})_{3}$-Restes zu verstehen, die mit Geometrieänderungen und Ladungsverschiebungen im Ringliganden gekoppelt ist. Wahrscheinlich stellt die energiereichere $\eta^{4}$-Struktur den Übergangszustand zwischen je $2 \eta^{3}$-Spezies dar.

Über die interessanten Konsequenzen der vorstehenden Resultate im Zusammenhang mit dem unterschiedlichen fluktionellen Verhalten von zu 1 heteroanalogen Systemen $15[20,21]$ und von Cycloheptatrien-eisentricarbonylen 16 [4a, 22] sowie über den Einfluß von Ringsubstituenten $\mathbf{R}$ in $\left[\mathrm{C}_{7} \mathrm{H}_{6} \mathrm{RFe}(\mathrm{CO})_{3}\right]^{-}$-Anionen $[3 \mathrm{a}, \mathrm{b}, \mathrm{c}]$ wird getrennt berichtet werden. 


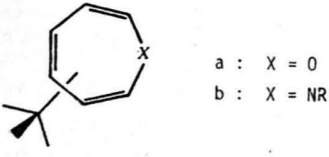

15

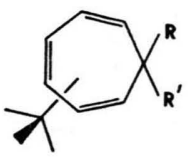

16

a : $R=R^{\circ}=H$

b : $R=H, R^{-}=$SiMe $_{3}, \mathrm{Gelle}_{3} \ldots$

\section{Anhang}

Die durchgeführten MO-Berechnungen sind vom Extended Hückel Typ [23]. Die verwendeten Parameter sind Tab. II zu entnehmen, wobei die $\mathrm{H}_{\mathbf{i i}}$ Werte für das Übergangsmetall aus einer SCCCRechnung mit quadratischer Ladungsiteration [24] an $\mathrm{C}_{4} \mathrm{H}_{6} \mathrm{Fe}(\mathrm{CO})_{3}$ mit experimenteller Geometrie erhalten wurden. Die Parameter für die $4 \mathrm{~s}$ - und $4 \mathrm{p}$ (single Slater AO's) und die 3d(double- $\xi$ )-Funktionen am Eisen entstammen der Literatur [25, 26]. Zur Ermittlung der Nichtdiagonalelemente $\mathbf{H}_{\mathbf{i j}}$ wurde eine modifizierte Wolfsberg-Helmholz-Beziehung verwendet [27].
Tab. II. Verwendete EH-Parameter.

\begin{tabular}{lllllll}
\hline & Atom/Orbital & $\mathrm{H}_{\mathrm{ii}}[\mathrm{eV}]$ & $\xi_{1}$ & $\xi_{2}$ & $\mathrm{C}_{1}{ }^{\mathrm{a}}$ & $\mathrm{C}_{2}{ }^{\mathrm{a}}$ \\
\hline $\mathrm{Fe}$ & $3 \mathrm{~d}$ & $-12,70$ & 5,35 & 1,80 & 0,5360 & $\mathbf{0 , 6 6 7 8}$ \\
& $4 \mathrm{~s}$ & $-9,17$ & 1,90 & & & \\
$\mathrm{C}$ & $\mathbf{4}$ & $-5,37$ & 1,90 & & & \\
& $2 \mathrm{~s}$ & $-21,40$ & 1,625 & & & \\
$\mathrm{O}$ & $2 \mathrm{p}$ & $-11,40$ & 1,625 & & & \\
& $2 \mathrm{p}$ & $-32,30$ & 2,275 & & & \\
$\mathrm{H}$ & $1 \mathrm{~s}$ & $-14,80$ & $\mathbf{2 , 2 7 5}$ & & & \\
\hline
\end{tabular}

a Koeffizienten der double- $\xi$-Funktionen.

Der Deutschen Forschungsgemeinschaft danke ich für die Unterstützung dieser Arbeit. [1a] H. J. Dauben und M. R. Rifi, J. Am. Chem. Soc. 85, 3041 (1963).

[1 b] W. v. E. Doering und P. P. Gaspar, J. Am. Chem. Soc. 85, 3043 (1963).

[1c] M. J. S. Dewar und N. Trinajstic, Tetrahedron 26, 4269 (1970).

[2] A. J. Birch und I. D. Jenkins, in H. Alper (Herausg.): Transition Metal Organometallics in Organic Synthesis, S. 55ff., Vol. 1, Academic Press, New York 1976.

[3a] H. Maltz und B. A. Kelly, Chem. Commun. 1971, 1390 (Li-Salz).

[3b] H. Knöchel, Diss. Universität Erlangen-Nürnberg 1972.

[3c] R. Kellner, Diss. Universität Erlangen-Nürnberg 1975.

[3d] H. Behrens, K. Geibel, R. Kellner, M. Moll und P. Würstl, VII. Internat. Conf. on Organomet. Chem., Abstracts of Papers 1975, 30 .

[3e] M. Moll, H. Behrens, R. Kellner, H. Knöchel und P. Würstl, Z. Naturforsch. 31 b, 1019 (1976).

[4a] H. Behrens, K. Geibel, R. Kellner, H. Knöchel, M. Moll und E. Sepp, Z. Naturforsch. 31 b, 1021 (1976).

[4b] G. Deganello, T. Boschi und L. Toniolo, J. Organomet. Chem. 97, C 46 (1975).

[4c] J. Takats und J. G. Reuvers, 2nd ACS-CIC Joint Conference, Juni 1977, Abstracts of Papers, INORG 090, Montreal, Kanada.

[4d] M. J. Bennet, J. L. Pratt, K. A. Simpson, L. K. K. Li Shing Man und J. Takats, J. Am. Chem. Soc. 98, 4810 (1976).

[5] Siehe nachstehende Abhandlung.

[6] F. A. Cotton und D. L. Hunter, J. Am. Chem. Soc. 98, 1413 (1976).

[7] M. A. Bennet, R. Bramley und R. Watt, J. Am. Chem. Soc. 91, 3089 (1969).
[8] Zum Ausdruck ,haptotrop“ vgl. Nguyen Trong Anh, M. Elian und R. Hoffmann, J. Am. Chem. Soc. 100, 110 (1978).

[9] G. Deganello et al. (s. Lit. 4) postulieren zwar ein Gleichgewicht zwischen 2 möglichen Formen von 1, gehen aber auf die Problematik nicht weiter ein.

[10] T. H. Whitesides und R. Budnik, Inorg. Chem. 15, 874 (1976).

[11] B. Dickens und W. N. Lipscomb, J. Chem. Phys. 37, 2084 (I962).

[12] R. Seip, Acta Chem. Scand. 26, 1966 (1972).

[13] T. A. Albright, P. Hofmann und R. Hoffmann, J. Am. Chem. Soc. 99, 7546 (1977).

[14a] M. Elian und R. Hoffmann, Inorg. Chem. 14, 1058 (1975).

[14b] M. Elian, M. M. L. Chen, D. M. P. Mingos und R. Hoffmann, Inorg. Chem. 15, 1148 (1976).

[15] E. Heilbronner und H. Bock, Das HMO-Modell und seine Anwendung, Verlag Chemie, Weinheim 1968.

[16] Es werden der Kürze halber die Bezeichnungen $\mathrm{s}, \mathrm{x}, \mathrm{y}, \mathrm{x}^{2}-\mathrm{y}^{2}, \mathrm{z}^{2} \ldots$ usw. statt $4 \mathrm{~s}, 4 \mathrm{p}_{\mathrm{x}}, 4 \mathrm{p}_{\mathrm{y}}, 4 \mathrm{p}_{\mathrm{z}}$, $3 \mathrm{~d}_{\mathrm{x}^{2}-\mathrm{y}^{2}}, 3 \mathrm{dz}^{2} \ldots$ usw. verwendet.

[17] F. A. Cotton, D. L. Hunter und P. Lahuerta, J. Am. Chem. Soc. 96, 4723 (1974).

[18] Theoretische Untersuchungen hierzu sind im Gange, Herrn Prof. R. Hoffmann, Cornell University, USA, danke ich für Informationen hierzu.

[19] G. Allegra und V. Perego, Ricerca Sci. 31(II-A), 362 (1961).

[20] R. Aumann, H. Averbeck und C. Krüger, Chem. Ber. 108, 3336 (1975).

[21] H. Günther und R. Wenzel, Tetrahedron Lett. 42, 4155 (1967).

[22 a] R. Wenzel, Dissertation, Köln 1969.

[22 b] L. K. K. Li Shing Man und J. Takats, J. Organomet. Chem. 117, C 104 (I976). 
[23] R. Hoffmann, J. Chem. Phys. 39, 1397 (1963); R. Hoffmann und W. N. Lipscomb, J. Chem. Phys. 36, 3179, 3489 (1962); 37, 2872 (1962).

[24] H. Basch, A. Viste und H. B. Gray, Theor. Chim. Acta 3, 458 (1965).

[25] R. H. Summerville und R. Hoffmann, J. Am. Chem. Soc. 98, 7240 (1976).
[26] J. W. Richardson, W. C. Nieuwpoort, R. R. Powell und W. F. Edgell, J. Chem. Phys. 36, 1057 (1962).

[27a] J. H. Ammeter, H.-B. Bürgi, J. C. Thibeault und R. Hoffmann, J. Am. Chem. Soc., im Druck.

[27 b] R. Hoffmann und P. Hofmann, J. Am. Chem. Soc. 98, 598 (1976). 